\title{
A case of early neonate tuberculosis in a three weeks old calf in Ethiopia
}

\author{
Getnet Mekonnen ${ }^{1}$, Balako Gumi ${ }^{2}$, Stefan Berg ${ }^{3}$, Andrew Conlan ${ }^{4}$, Martin Vordermeier ${ }^{3}$, \\ Gobena Ameni ${ }^{5}$, and James Wood ${ }^{6}$ \\ ${ }^{1}$ Affiliation not available \\ ${ }^{2}$ Addis Ababa University \\ ${ }^{3}$ Animal and Plant Health Agency \\ ${ }^{4}$ University of Cambridge School of Clinical Medicine \\ ${ }^{5}$ Akilu Lemma Institute of Pathology, Addis Ababa University, P.O.Box 1176, Addis \\ Ababa, Ethiopia. \\ ${ }^{6}$ University of Cambridge
}

April 29, 2020

\begin{abstract}
Bovine tuberculosis is a chronic disease rarely observed in an early age. Our observation of tubercle lesions in the lungs of a three week old calf and confirmation of Mycobacterium bovis strains illustrate that the progression of tuberculosis in neonates can be rapid under natural conditions, contributing to transmission within-herds.
\end{abstract}

\section{Key Clinical Message}

This report illustrates that calves may be infected with bovine tuberculosis at early age under natural conditions and progression can be rapid. Thus, testing of calves need to be considered in any control program to reduce the risk of transmission.

\section{INTRODUCTION}

Bovine tuberculosis (bTB) is a chronic disease, caused primarily by $M$. bovis, affecting cattle, other domesticated animals, certain wildlife species, non-human primates, and humans. ${ }^{1-3}$ Cattle can be infected at any age, but tubercle lesion development and subsequent clinical signs may appear at a later stages. Calves can be born with tuberculosis congenitally when infected with $M$. boviseither via the umbilical vein from an infected genital tract of the dam, from the infected placenta, or from infected amniotic fluid. ${ }^{4}$ After birth, neonates can also be infected even at an early stages following delivery, mainly by inhaling infected droplets and/ or by ingesting raw milk or colostrum from infected cows. Other routes of infection such as cutaneous, though not common, are still important in situations where the prevalence of bTB is high. ${ }^{5}$ As the course of the disease is slow compared to many other infectious diseases, infected animals can spread bTB to other herd mates and newborns before it begins to manifest clinical signs. The transmission rate may be faster at late stages of infection as the number of organisms being shed is larger in this stage. ${ }^{1}$

The observation of characteristic tubercle lesions in the lungs of a three weeks old male calf led us to raise questions like 'how the calf could get the infection?' and 'how fast is the disease progression under natural 
infection?'. In order to get answers to these questions, further investigation was made and this case report was compiled from the pathological and molecular investigations conducted for the diagnosis of the case.

\section{CASE HISTORY}

A dairy farm located in northern Ethiopia and with a herd size of 100 cattle (86 crossbreeds of HolsteinFrisian and Zebu breeds and 14 Zebu breeds) was screened for bTB by the SICCT test. As it was learned from its history, the farm was newly established just a year earlier through the purchase of cows and heifers from dairy farms and markets in Mekelle City and nearby towns, including Adigrat and Humera. The SICCT testing was conducted on the herd in 2016 by Mekonnen et al. ${ }^{6}$ and a prevalence of $12 \%$ (95\% CI: 7 to $19.8 \%$ ) was recorded. One of the reactors was a three weeks old calf (ID 8A0597) and its skin induration was $8 \mathrm{~mm}$ according to the calculation of the SICCT test result. All positive reactors (including the calf) were removed from the herd following disclosure of the test results. It was observed from the husbandry of the farm that all calves in the farm were sharing the same housing with the adults. Moreover, the calves were fed on pooled milk obtained from milking cows in the herd including one SICCT reactor cow, and looked after by the same individuals who were looking after the adult cattle.

The dam of the calf (ID 8A0417) was a 10 years old cross of Holstein-Frisian and Zebu breeds. The body condition of this cow was rated as 'Good' and it was negative for bTB by the SICCT test according to the interpretation recommended by OIE. ${ }^{7}$ In addition, nasal discharge was collected from the dam using a cotton swab designed for such purpose, and the PBS wash of the swab was centrifuged at $4000 \mathrm{rpm}$ for $15 \mathrm{~min}$. Genomic DNA was extracted from the pellet using a Qiagen DNA extraction kit as per the manufacturer's instruction, ${ }^{8}$ and the eluent tested by an RD4-based PCR assay for specific identification of $M$. bovis. The primers defined by Brosch et al. ${ }^{9}$ were used in the PCR assay procedure described by Berg et al . ${ }^{10}$

\section{INVESTIGATION}

The body condition of the calf was moderate, but it was characterised with some hair loss on various parts of its body and appeared depressed. In post-mortem, multiple granulomas were observed in the apical lobe of the lungs (FIGURE 1). A single $\sim 20 \mathrm{~mm}$ diameter of a loosely encapsulated granuloma-like structure was also observed in the diaphragmatic lobe. Lymph nodes and other vital organs appear normal.

\section{(FIGURE 1 here)}

The culturing result (Löwenstein-Jensen medium slant) from lung tubercles of the calf showed two strains of typical $M$. boviscolony morphology. Heat-killed colonies were tested by RD4-based PCR and confirmed that both strains were $M$. bovis . Spoligotyping ${ }^{11}$ then classified these two strains into different spoligotype patterns, SB0134 and SB0133 (FIGURE 2). Genomic DNA from the dam's nasal discharge was positive for $M$. bovis by the RD4-based PCR assay; however, spoligotyping was not sensitivity enough to confirm the pattern.

\section{(FIGURE 2 here)}

\section{DISCUSSION}

M. bovis strains identified in the present case, classified as spoligotypes SB0134 and SB0133, suggesting mixed infection. Although mixed infection has not been frequently described in Ethiopia, it is more likely to happen in herds which have established their stocks from different farms, as was the case for the present herd. Hence, the mixed infection observed in this calf could be due to transmission from more than one reactor, possibly recruited from different herds without prior testing for bTB.

The time-frame for development of tubercle lesions in the investigated calf is comparable with artificially infected calves via respiratory route under experimental conditions. ${ }^{12}$ However, under such conditions; the extent of pathology could vary depending on the infective dose and factors specific to the animals and husbandry systems. Interestingly, the gross pathological lesions in the present calf were found only in specific lobes of the lungs. 
Evidences have shown that the route of transmission of $M$. boviscould be inferred from lesions distribution. ${ }^{13}$ In the present calf, tubercle lesions were localized in the lungs and no tubercle lesions were observed in other tissues and organs, which could suggest inhalation of infectious droplets as a possible route of infection while ingestion of infected colostrum or milk might seem less likely. Other possibilities of in-utero transmission through hematogenous route and/or aspiration of $M$. bovis contaminated amniotic fluid ${ }^{14,15}$ could not be justified as there was no lesion suggestive of bTB in other vital organs, such as liver. Tuberculosis transmitted hematogenously is typically characterised by presence of hepatic tubercles or more disseminated form of the disease ${ }^{16}$ while through aspiration of infected amniotic fluid, the primary tubercle could be in the lungs and/or the gut.

The molecular test result imply that the dam was infected with $M$. bovis even though it was not a reactor to the SICCT test (the increase at the PPD-B injection site was $3.2 \mathrm{~mm}$ while at the PPD-A site it was $2.9 \mathrm{~mm}$ ). This could potentially be due to a development of anergic status, in which the dam would either had been be in a late disease stage or immunosuppressed attributable to the hormonal effects during preand post-parturition. An animal with a chronic stage of the disease is known to be highly infectious and would likely serve as a good source of infection to a neonate by transmission in several possible ways. ${ }^{17,}{ }^{18}$ Therefore, it is highly likely that the calf in this observed case got infected in its first week of life, while suckling the colostrum by inhalation of aerosol primarily generated from its dam, although the possibility of transmission from other infected animals could not be ruled out as all reactors in this farm shared the same housing and husbandry system as the calf. In fact, the latter could explain why strains of the two spoligotype patterns were isolated from its lungs.

In conclusion, although this is a single case, it illustrates that the progression of bTB in calves can be rapid under natural conditions, contributing to transmission within herds. This has important implications in the appropriate structure of models used to estimate the rate of transmission within herds. ${ }^{19}$ Calf can respond to the tuberculin test at a very early age, and thus should be considered in the routine bTB screening to reduce transmission within herds.

\section{AUTHORS' CONTRIBUTIONS}

GAM performed the investigation, analyzed the data, and wrote the report. BG, SB, AJKC, MV, GA, and JLNW contributed to the study conception and design and reviewed the report.

\section{ACKNOWLEDGEMENTS}

This work was funded by the Biotechnology and Biological Sciences Research Council, the Department for International Development, the Economic \& Social Research Council, the Medical Research Council, the Natural Environment Research Council and the Defence Science \& Technology Laboratory, under the Zoonoses and Emerging Livestock Systems (ZELS) program, ref: BB/L018977/1.

\section{COMPETING INTEREST}

The authors declare that they have no competing interests.

\section{ETHICAL CONSIDERATIONS}

This study was under the framework of a research protocol approved by the Institutional Review Board (IRB) of Aklilu Lemma Institute of Pathobiology, Addis Ababa University (Reference numberIRB/ALIPB/2018).

\section{AVAILABILITY OF DATA AND MATERIALS}

All data generated and / or analyzed during this study are not publicly available but are available from the corresponding author on reasonable request.

\section{REFERENCES}

1. CFSPH. 2009. Bovine tuberculosis Fact sheet. Center for Food Security and Public Health (CFSPH), Iowa State University. 
2. Cousins DV. 2001.Mycobacterium bovis infection and control in domestic livestock.Rev Sci Tech Off Int Epiz 20:71-85.

3. More S, Bøtner A, Butterworth A, Calistri P, Depner K, Edwards S, et al. 2017. Assessment of listing and categorization of animal diseases within the framework of the Animal Health Law (Regulation (EU) No 2016/429): bovine tuberculosis.EFSA - DOPTED: 30 June 2017. doi: 10.2903/j.efsa.2017.4959

4. Ozyigit MO, Senturk S, Akkoc A. 2007. Suspected congenital generalised tuberculosis in a newborn calf. Vet Rec 160: 307-308.

5. Phillips CJ, Foster CR, Morris PA, Teverson R. 2003. The transmission of Mycobacterium bovis infection to cattle. Res Vet Sci 74: 1-15.

6. Mekonnen GA, Conlan AJK, Berg S, Ayele BT, Alemu A, Guta S, et al. 2019. Prevalence of bovine tuberculosis and its associated risk factors in the emerging dairy belts of regional cities in Ethiopia. Prev Vet Med 168: 81-89.

7. OIE. 2009. Bovine tuberculosis. OIE-manual of diagnostic tests and vaccines for terrestrial animals. Paris.

8. Qiagen. 2016. QIAamp DNA Mini and Blood Mini Handbook. 5th Edition. Sample and Assay Technologies. Available: http://www.qiagen.com (accessed 30 March 2019).

9. Brosch R, Gordon SV, Marmiesse M, Brodin P, Buchrieser C, Eiglmeier K, et al. 2002. A new evolutionary scenario for the Mycobacterium tuberculosis complex. PNAS - USA99: 3684-3689.

10. Berg S, Firdessa R, Habtamu M, Gadisa E, Mengistu A, Yamuah L, et al. 2009. The burden of mycobacterial disease in Ethiopian cattle: implications for public health. PLoS ONE 4. doi: 10.1371/journal.pone.0005068.

11. Kamerbeek J, Schouls L, Kolk A, van Agterveld M, van Soolingen D, Kuijper S, et al. 1997. Simultaneous detection and strain differentiation of Mycobacterium tuberculosis for diagnosis and epidemiology. $J$ Clin Microbiol 35: 907-914.

12. Kao RR, Gravenor MB, Charleston B, Hope JC, Martin MHoward CJ. 2007. Mycobacterium bovis shedding patterns from experimentally infected calves and the effect of concurrent infection with bovine viral diarrhoea virus. $J$ R Soc Interface 4: 545-551.

13. Francis, J. 1971. Susceptibility to tuberculosis and the route of infection. Aust Vet J 47: p. 414.

14. Vural SA, Tunca R. 2001. Generalized tuberculosis in a 45 day-old calf. Dtsch Tierarztl Wochenschr 108: 468-470.

15. Espejel MdC, Del Rio JC, Medrano A, Santillan MA, Morales JF. 2018. Congenital tuberculosis in a 25-day-old female calf. Turk J Vet Anim Sci 42: 135-138.

16. Tomar RPS, Gupta A, Prasad TR, Bhalla PJS, Murthy GSN. 2008. Congenital Tuberculosis. Med J Armed Forces India 64: 78-80.

17. Raj P, Sarin YK. 2014. Congenital Tuberculosis in a Neonate: A Diagnostic Dilemma. J Neonat Surg 3: 49.

18. Yeh J-J, Lin S-C, Lin W-C. 2019. Congenital tuberculosis in a neonate: A case report and literature review. Front Pediatr $7: 255$.

19. Conlan AJK, McKinley TJ, Karolemeas K, Pollock EB, Goodchild AV, Mitchell AP, et al. 2012. Estimating the Hidden Burden of Bovine Tuberculosis in Great Britain.PLoS Comput Biol 8: e1002730. 

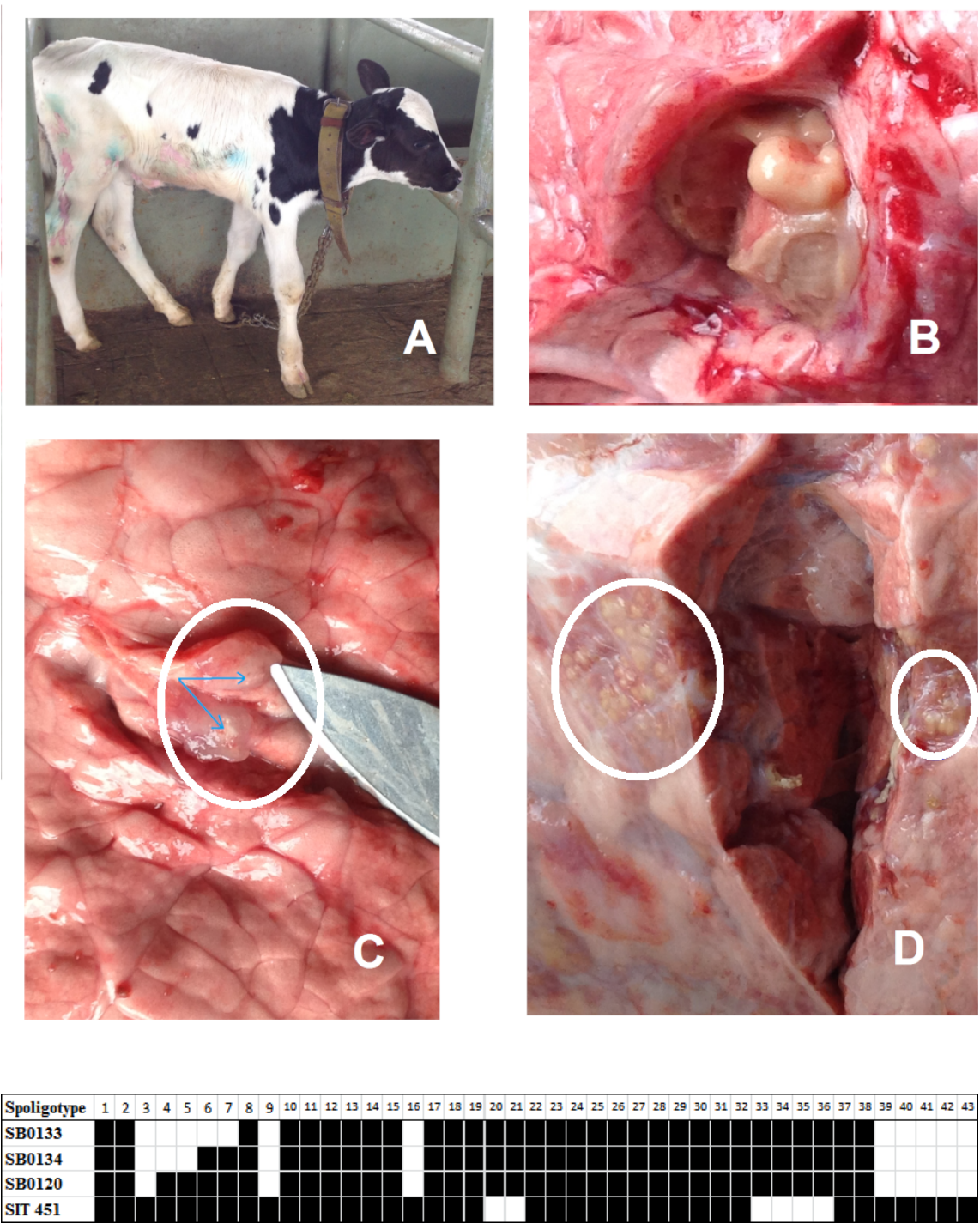